\title{
Servicios bibliotecarios para dispositivos móviles: El caso de las bibliotecas vinculadas al Consejo Nacional para Asuntos Bibliotecarios de las Instituciones de Educación Superior de México
}

\author{
Eugenia de los Ángeles Ortega Martínez \\ Universidad Nacional Autónoma de México - UNAM, México
}

CASE REPORT

\begin{abstract}
Resumen
Objetivo: Analizar cuáles son los servicios bibliotecarios y las aplicaciones (apps) que se están utilizando dentro de las bibliotecas académicas de las Instituciones de Educación Superior adscritas al Consejo Nacional para Asuntos Bibliotecarios de las Instituciones de Educación Superior (CONPAB-IES, A. C.) de México. Método: Se realizó una revisión de la literatura en bases de datos y revistas especializadas en Bibliotecología y Ciencias de la Información con ayuda de los recursos digitales de la Dirección General de Bibliotecas de la Universidad Nacional Autónoma de México. Resultados y conclusiones: El desarrollo de apps y servicios bibliotecarios dispositivos móviles en bibliotecas académicas de las Instituciones de Educación Superior, adscritas al CONPAB-IES, A. C., muestra una tendencia por el desarrollo de colecciones digitales en formatos amigables para PC y laptop, a diferencia de smartphones y tablets.
\end{abstract}

Palabras clave

Dispositivos móviles ; Bibliotecas académicas ; Servicios bibliotecarios ; México

\section{Library services for mobile devices: The case of academic libraries affiliated to National Council for Library Affairs Higher Education Institutions of México}

\begin{abstract}
Objective: The aim of this paper is to analyze library services and its applications (apps) that are being used within academic libraries affiliated to the National Council for Library Affairs Higher Education Institutions AC (CONPAB-IES, AC) in Mexico. Method: It has been made a literature review with databases and journals specialized in Library and Information Sciences through the Digital Resources obtained of the General Management of Libraries (DGB) of the National Autonomous University of Mexico (UNAM). Results and conclusions: The development of applications (apps) library services and mobile devices in academic libraries in Institutions of Higher Education, linked to CONPAB - IES, A.C., shows the library services within academic libraries and the preference for the development of resources of the digital collections and networks through their websites in friendly formats for PC and laptop, instead of smartphones and tablets.
\end{abstract}

Keywords

Mobile devices ; Academic libraries ; Library services ; Mexico

\section{Introducción}

Desde la invención de los dispositivos móviles, la mercadotecnia en la "web móvil" se ha convertido en un espacio imprescindible para cualquier proveedor de productos y servicios. Según datos del Banco Mundial ${ }^{1}$, en los Estados Unidos se registró que del año 2011 al 2015; de cada 100 habitantes, 98 personas pagaron por servicios de telefonía. En México esta cifra es de 83 personas por cada 100 habitantes, de los cuales sólo 16.71 son suscripciones telefónicas fijas ${ }^{2}$. Expertos determinan que para el año 2020 cerca del $62 \%$ del acceso a internet, será móvil ${ }^{3}$. 
En el contexto de las bibliotecas académicas, la web móvil poco a poco se ha convertido en la protagonista de numerosas publicaciones sobre su uso y aplicaciones para los servicios bibliotecarios. En este sentido, es interesante resaltar que en los últimos cinco años (2010-2015) las bases de datos: INFOBILA, TESIUNAM, LIBRUNAM, HERMES-Open Access, Library and Information Science Abstracts (LISA), Emerald, así el Consorcio Nacional de Recursos de Información Científica y Tecnológica (CONRICYT) y el Catálogo Nacional de Bibliotecas Académicas de la Dirección General de Bibliotecas de la Universidad Nacional Autónoma de México, han publicado 1,125 títulos de artículos y libros que involucran directamente las palabras clave: library services, library services, mobile devices, academic libraries y México, con sus respectivos términos en español.

Como se puede observar en la Grafica 1, el 63\% de los artículos y libros son publicaciones administradas por el CONRICYT y de ellas, solamente el $5.2 \%$ se refieren al contexto de las bibliotecas académicas en México. A diferencia de CONRICYT, los resultados obtenidos en la base de datos LISA, el $62 \%$ de los títulos fueron en inglés y el resto en español; el $70 \%$ de este contenido son publicaciones relacionadas con las bibliotecas académicas de los Estados Unidos de los cuales el $80 \%$ muestra una notable preferencia por los usuarios hacia servicios bibliotecarios para smartphones.

Gráfico 1 - Publicaciones sobre servicios bibliotecarios para dispositivos móviles en bibliotecas académicas (2011-2015).

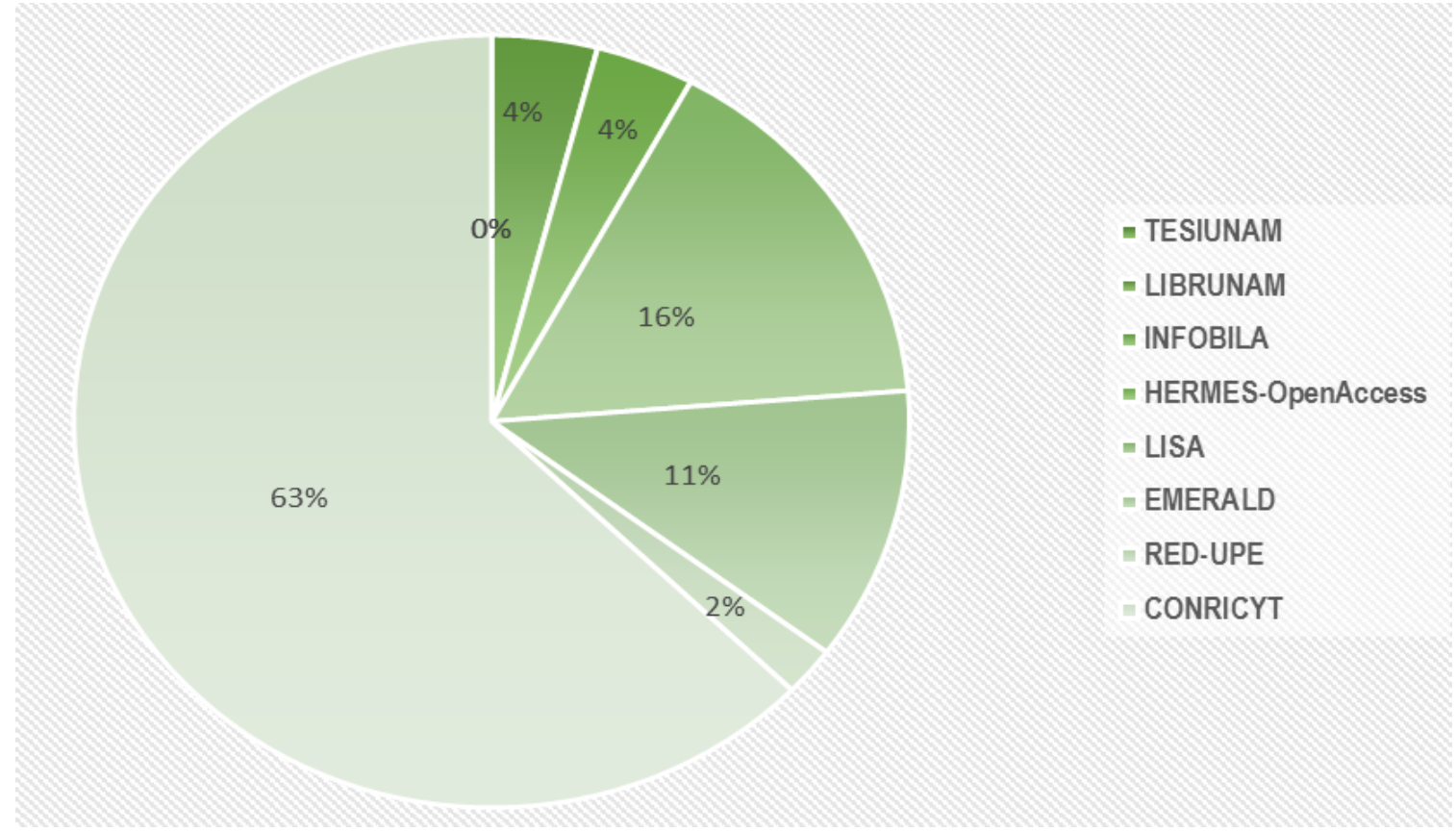

\section{Servicios bibliotecarios para dispositivos móviles.}

Los servicios bibliotecarios, son las actividades orientadas a satisfacer necesidades específicas de los usuarios mediante el préstamo en sala, a domicilio e inter-bibliotecario; la consulta; el fotocopiado de materiales susceptibles de serlo; el acceso a Internet; y la disposición de instalaciones para fines diversos, relacionados con el desarrollo educativo, cultural y social de la población ${ }^{4}$. Con la aparición de los dispositivos móviles, la versatilidad de los servicios bibliotecarios han re-planteado aspectos relacionados con su implementación, uso, manejo, organización y acceso de la información a través de la nube (Cloud), a una escala globlal (Webscale).

En los últimos años la web móvil ha crecido exponencialmente y como lo reporta el Centro de Cómputo Bibliotecario en Línea (OCLC) las particularidades de este tipo de actividades es que tienen el potencial de incrementar la visibilidad, generar colecciones colectivas y crear redes de colaboración a nivel global ${ }^{5}$. En este 
trabajo se dio prioridad a los teléfonos inteligentes (smartphones) y tabletas (tablet computer), debido a la popularidad y uso para la consulta y acceso a la información de los usuarios en las bibliotecas académicas.

Los smartphones son teléfonos celulares con una plataforma informática móvil capaz de almacenar datos y realizar actividades semejantes a las de una minicomputadora; una característica indispensable de estos dispositivos es el acceso al correo electrónico ${ }^{6}$. Por su parte, la Tablet, es una computadora portátil de mayor tamaño que un teléfono inteligente y con pantalla completamente táctil ${ }^{7}$.

Los servicios bibliotecarios diseñados para la web móvil tienen un impacto masivo y al mismo tiempo individualizado. Este tipo de aplicaciones son cada vez más populares entre la comunidad universitaria y

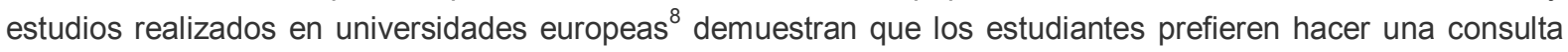
del catálogo en su smartphone, en lugar de ir directamente a la página web de la biblioteca. Es importante señalar que la limitación principal en esta modalidad es que está sujeto a la conectividad de internet wi-fi .

\section{Servicios bibliotecarios para dispositivos móviles en el contexto de las bibliotecas académicas.}

Las bibliotecas académicas son un espacio privilegiado porque cuentan con diversos factores tecnológicos y de investigación que les permiten innovarse constantemente. Estos espacios albergan información vital para la comunidad universitaria y la investigación científica; por ello el acceso a las bases de datos, catálogos en línea, así como los servicios de internet dentro de la biblioteca, fueron parte de las innovaciones prioritarias a principios del siglo XXI. No obstante, diversos autores como Kae Perng ${ }^{10}$, Graham Walton ${ }^{11}$, señalan que una parte vital de los servicios de información en el futuro cercano será el desarrollo de aplicaciones (apps) para dispositivos móviles.

En el año 2011, Aiugo Li publicó que de 39 universidades en China, exceptuando las que están en las regiones de Hong Kong, Taiwan y Macao, solamente el 36\% aplicaban servicios móviles para sus bibliotecas; Por su parte Catharine Bomhold realizó un análisis de 73 universidades públicas de los Estados Unidos, concluyendo que en el año 2010 sólo $26.1 \%$ de las instituciones ofrecían servicios móviles para sus usuarios, pero esta cantidad se incrementó considerablemente hasta un 70\% para el año 2013.

Como se observa, este tipo de tecnología tiene un crecimiento acelerado dentro de las bibliotecas académicas, sobre todo en los últimos cinco años. No obstante, Joan Lippincot ${ }^{12}$ puntualiza que aunque la demanda en este tipo de servicios es notable y se están creando numerosas aplicaciones para los servicios bibliotecarios, aún existen evidencias que señalan el rezago en su aprovechamiento.

Por ejemplo en la Universidad de Boston en los Estados Unidos y la Universidad de Salamanca en España, utilizan algunas apps como administradores de referencias bibliográficas Zotero, Mendeley, RSS, QR-Codes, mismas que no fueron diseñadas por la propia universidad pero que resultaron efectivas para parte de sus actividades; sin embargo, aunado a lo que menciona Lippincot existen poco más de 45 apps para realizar otros servicios dentro de la biblioteca como: impulsar y organizar el trabajo, para lectura y noticieros ${ }^{13}$.

Parte de la problemática relacionada con la falta de aprovechamiento de los servicios bibliotecarios a través de la web móvil en México, es que el $63 \%$ de los mexicanos entre los 13 a los 44 años de vida productiva, invierten en promedio 6 horas diarias navegando por la web y que de este tiempo el $85 \%$ va directamente a la consulta y uso de sus redes sociales ${ }^{14}$. Aunado a ello, también existe desconfianza del profesorado en las herramientas tecnológico-educativas desarrolladas por las universidades y las bibliotecas como apoyo a sus labores docentes.

En un estudio realizado en la Facultad de Ingeniería de la UNAM en el año 2011, el 64\% del profesorado muestra que hace uso de los medios tecnológicos como apoyo a sus cursos, pero solamente $19 \%$ tiene un sitio web docente y expresan que el uso de este tipo de tecnología no les genera confianza ya que en parte genera pasividad en los estudiantes y limita sus intereses por algo más allá que no esté en la bibliografía o materiales del curso ${ }^{15}$. Aunque aún no existe un estudio que correlacione la falta de aprovechamiento de los servicios bibliotecarios con la web móvil y la falta de confianza o habilidades informativas del profesorado en las IES de México, como primer paso es necesario saber qué es lo que dice la literatura publicada y sus propias páginas web. 


\section{Metodología}

Debido a que el contexto de las universidades mexicanas es muy amplio y a su vez cada sistema de bibliotecas o centros de información están delimitados por las políticas internas de las instituciones, el primer paso en este estudio fue delimitar el universo de estudio hacia las IES en México afiliadas al CONPAB-IES, A.C. Este criterio de selección se dio en parte porque existen diagnósticos previos que han sido publicados hasta el año 201 y esto representa un avance significativo en el contexto del estudio; es importante señalar que esta investigación aportará nuevos datos y conocimiento en el tema ${ }^{16}$.

La revisión de la literatura se realizó con bases de datos y revistas especializadas en Bibliotecología y Ciencias de la Información adscritos a los recursos digitales de la Dirección General de Bibliotecas de la Universidad Nacional Autónoma de México. Los términos utilizados para la búsqueda fueron: Servicios de Información, Dispositivos Móviles, Bibliotecas académicas y México. Además, la delimitación de las publicaciones tuvo un rango específico (2011-2015).

De manera complementaria se revisaron las páginas web de las bibliotecas académicas de 34 IES, de las cuales 30 tienen un apartado fácil de localizar visualmente, pero en las 4 restantes fue necesario ingresar al mapa del sitio web para poder identificar la palabra "biblioteca" ó "servicios bibliotecarios". Los resultados obtenidos se describen a continuación.

\section{Análisis de los resultados}

En el caso de las bibliotecas académicas en México, se observa que existe un importante desarrollo en colecciones y bibliotecas digitales, pero existe poca información para poder determinar cómo se están implementando los servicios bibliotecarios para la web móvil. Al respecto, en el análisis de la literatura sólo se encontraron dos apps (FIME Móvil de la Universidad Autónoma de Nuevo León y Mi Tec Móvil del Tecnológico de Monterrey), pero estas aplicaciones son un registro para que los alumnos registrados en las universidades puedan acceder a los servicios de la biblioteca y además a otro tipo de trámites administrativos ${ }^{17}$.

En el análisis de las páginas web (Tabla 1), se observa que los servicios bibliotecarios que pueden obtener los usuarios que acuden a las instalaciones físicamente son: préstamo interno, externo, inter bibliotecario, así como trámites administrativos para los estudiantes: carta de no adeudo de materiales. Existen además otro tipo de actividades relacionadas con la formación de usuarios: visitas guiadas, cursos de bases de datos, entre otros. Sin embargo, no se publican apps para cualquiera de ellos y existe la posibilidad de que se cuente con ellos pero que se tenga algún tipo de exclusividad.

Respecto a los recursos digitales de las instituciones, se encontró que el acceso a internet es básico en el $90 \%$ de los casos y todas las páginas web de las universidades tienen catálogo en línea; en el $80 \%$ de los sitios sus bases datos se encuentran adscritos a sus bibliotecas digitales, con autorización restringida. Aunado a lo anterior, no se encontraron publicadas aplicaciones específicas para la biblioteca o los servicios bibliotecarios, pero en redes sociales se encontró que el $60 \%$ de las bibliotecas cuentan con registro en Twitter y Facebook y el $11 \%$ anuncia tutoriales y otro tipo de contenidos en Youtube.

Finalmente es importante mencionar que se publican otros proyectos interesantes en el contexto de colecciones digitales, cursos en línea, Desarrollo de Habilidades Informativas (DHI), referencias virtuales, chats en línea, servicios para débiles visuales, entre otros, todo dentro de los recursos digitales de las propias instituciones.

\section{Conclusiones}

Se infiere que los servicios bibliotecarios para la web móvil dentro de las bibliotecas académicas de las IES afiliadas al CONPAB-IES, A.C., son escasos y las páginas web de sus bibliotecas, brindan solamente un panorama general de los servicios bibliotecarios que se ofrecen a los usuarios, dentro y fuera de sus instalaciones. Existe una notoria prioridad por el desarrollo de colecciones y bibliotecas digitales, así como la creación de redes académicas y de investigación entre las universidades y la disponibilidad en sus acervos 
digitales (tesis, artículos, libros, entre otros) y de las bases de datos afiliadas; en particular, se refleja un importante avance en la creación de catálogos en línea colectivos.

Como se observa en la Tabla 1, los servicios publicados por las universidades varían pero en general ofrecen: catálogos en línea, uso de materiales audiovisuales y salas multimedia, así como los servicios tradicionales de consulta préstamos y salas de lectura. Existen además otro tipo de servicios que en esencia son similares a los que se ofertan en la propia biblioteca pero de manera virtual como lo son las referencias, asesoría a través de un chat en línea, renovaciones de libros y el apartado de documentos. Además, es muy probable que exista una falta de comunicación entre los servicios que se tienen en realidad y los que se publican, como es el caso de la conexión Wi-fi, que solo aparece disponible para los usuarios en cuatro de las 34 universidades consultadas.

Es importante señalar que en la antesala de los servicios de información para dispositivos móviles, aún no se publican aplicaciones (apps) exclusivas para las actividades de la biblioteca académica, además el diseño y formato de la mayoría de las páginas no es una versión que permita navegar amigablemente con dispositivos móviles, como se comprobó con la revisión realizada en este estudio y que quizá la tecnología avanza con pasos agigantados y de acuerdo a la revisión de la literatura y las tendencias en los servicios bibliotecarios para la web móvil, el desarrollo de aplicaciones se convertirá sin duda, en una de las actividades con grandes oportunidades para las bibliotecas académicas en México a corto plazo.

\section{Bibliografía}

[1] BANCO MUNDIAL, (2015) <<Abonos a teléfonos celulares (por cada 100 personas)〉>, Unión Internacional de Telecomunicaciones, Informe sobre el Desarrollo Mundial/TIC de las Telecomunicaciones y estimaciones del Banco Mundial, [Consultado: 2015-12-21], Disponible en: http://datos.bancomundial.org/indicador/IT.CEL.SETS.P2

[2] INSTITUTO FEDERAL DE TELECOMUNICACIONES, (2016), Número de suscripciones fijas por cada 100 habitantes, [Consultado: 2015-12-21]. Disponible en: http://siemt.ift.org.mx/

[3] DAVIDE, Strusani, (2015), Digital inclusión and mobile taxation in Mexico, London, Deloitte, [Consultado: 2016-01-03], Disponibleen: http://www.gsma.com/mobilefordevelopment/wp-content/uploads/2015/09/GSMA_Mexico-Report_WEB.pdf

[4] CÁMARA DE DIPUTADOS, CONGRESO DE LA UNIÓN, (AÑO) <<Ley General de Bibliotecas»>, Gaceta Parlamentaria, (8 enero 2014), [Consultado: 2014-05-21]. Disponible en: http://gaceta.diputados.gob.mx/Gaceta/62/2014/ene/20140108II.htm|\#Iniciativas

[5] ONLINE COMPUTER LIBRARY CENTER, INC, (2011), Libraries at webscale, a discussion document, Dublin, Ohio, [Consultado: 2015-12-21], Disponible en: https://www.oclc.org/reports/webscale.en.html

[6] ZIFF DAVIS, (2016), Tablet computer, Enciclopedya, [consultado: 2016-02-08], Disponible en: http://www.pcmag.com/encyclopedia/term/52520/tablet-computer

[7] ZIFF DAVIS, (2016), Smartphone, [consultado: 2016-02-08]. Disponible en: http://www.pcmag.com/encyclopedia/term/51537/smartphone

[8] VASSILAKAKI, Evgenia, (2014), <<Mobile information services in libraries: a review of current trends in delivering information>> Interlending \& Document Supply, 42 (4), 176-186. [Consultado: 2015-12-05], Disponible en: http://dx.doi.org/10.1108/LLDS-08-2014-0037

[9]PATERSON, Lorraine; LOW, Bonn, (2011), Student attitudes towards mobile library services for smartphones, Edimburg, UK, University of Edimburg, [Consultado: 2015-12-22]. Disponible en: www.emeraldinsight.com/0737-8831.htm, 29 (3), $412-$ 423, DOI: $10.1108 / 07378831111174387$.

[10] WONG, Kae Perng, (2013), <<Library services for mobile devices: the National Institute of Education Library experience >, Library HiTech News, [Consultado: 2016-01-03], Disponible en: http://dx.doi.org/10.1108/LHTN-09-20130048, 30(9): 7-11.

[11] WALTON, Graham, (2014), «<Will Smart Phones and Other Bring Your Own Devices (BYOD) Dominate How Academic Library Services are Developed and Delivered for the Foreseeable Future >, New Review of Academic Librarianship, 20:13, DOI: 10.1080/13614533.2014.875294 
[12] LIPPINCOTT, Joan K, (2010), $<<A$ mobile future for academic libraries $>$, Reference Services Review, Washington, DC, EE.UU, [Consultado: 2016-01-11]. Disponible en: http://dx.doi.org/10.1108/00907321011044981, 38(2): 205 - 213.

[13] S, Robert, (2014), <<45 Most exciting apps for librarians >>, Librarysciencelist.com, [consultado: 2016-01-04], Disponible en: http://librarysciencelist.com/author/roberts/

[14] ASOCIACIÓN MEXICANA DE INTERNET. (2015), 11vo. Estudio de los hábitos de los internautas mexicanos. México, [consultado: 2016-04-07]. Disponible en: https://www.amipci.org. mx/es/estudios

[15] VALDÉZ Y ALFARO, Irene Patricia, (2012), Encuesta para profesores sobre el uso de Tecnologías de Información y Comunicación, TIC's, México, UNAM, [Consultado: 2015-12-02]. Disponible en: http://dcb.fi- c.unam.mx/ProyectoTICS/ contenidos/encuestaDCB TICS-2012-1.pdf

[16] CONSEJO NACIONAL PARA ASUNTOS BIBLIOTECARIOS DE LAS INSTITUCIONES DE EDUCACIÓN SUPERIOR, A. C., (2015) <<¿Quiénes somos?»>, [Consultado: 2015-12-22]. Disponible en: http://www.conpab.org.mX/

[17] GABRIEL GUTIÉRREZ, Fernando, (2013), <<Aplicaciones móviles de bibliotecas de América Latina〉>, Infotecarios, Universidad Nacional de Luján, [Consultado: 2015-12-22]. Disponible en: http://www.infotecarios.com/aplicaciones-movilesde-bibliotecas-de-america-latinal 


\section{Apéndice}

Tabla 1 - Servicios bibliotecarios publicados en las páginas web de las IES que pertenecen a CONPABIES en 2016.

\begin{tabular}{|c|c|c|c|c|c|c|c|c|c|c|c|c|c|c|c|c|c|c|c|c|}
\hline INSTITUCIONES DE EDUCACION SUPERIOR & $\mathrm{CL}$ & $P$ & PMA & A & AD & B & $\mathrm{C}$ & AP & $\mathrm{DHI}$ & $\mathrm{R}$ & $\mathrm{BD}$ & DSI & IyD & $\mathrm{L}$ & FU & Lo & wifi & FU & ChL & $\mathrm{Al}$ \\
\hline Universidad Autónoma de Aguascalentes & $x$ & $x$ & $\mathrm{x}$ & $\mathrm{x}$ & & & & & $x$ & & & & & & $x$ & & $\mathrm{x}$ & $\mathrm{x}$ & & \\
\hline Universidad Autónoma de Baja Calfornia & $\mathrm{x}$ & $x$ & & $\mathrm{x}$ & & & & & & & & & & & & & $\mathrm{x}$ & & & \\
\hline Universidad Autónoma de Baja California Sur & $\mathrm{x}$ & & & & & & & & & & $\mathrm{x}$ & & & & $\mathrm{x}$ & & & & & \\
\hline Universidad Autónoma de Campeche & & $x$ & & & & & & & & & $x$ & & & & $\mathrm{x}$ & & & & & \\
\hline Universidad Autónoma del Carmen & & & & & & & & & & & & & & & & & & & & \\
\hline Universidad Autónoma de Coahuila & $\mathrm{x}$ & & & & & & & & & & $x$ & & & & & & & & & \\
\hline Universidad de Colima & $x$ & & $\mathrm{x}$ & & & & & & & & & & & & $\mathrm{x}$ & & & & $\mathrm{x}$ & \\
\hline Universidad Autónoma de Chiapas & $x$ & & & & & & & & & & $\mathrm{x}$ & & & & & & & & & \\
\hline Universidad Autónoma de Chihuahua & $x$ & $x$ & & & $x$ & & $\mathrm{x}$ & & & $x$ & $\mathrm{x}$ & & $x$ & & $\mathrm{x}$ & & & & & \\
\hline Universidad Autónoma de Ciudad Juárez & $\mathrm{x}$ & & & & & & & & & $\mathrm{x}$ & $\mathrm{x}$ & & $x$ & & $\mathrm{x}$ & & & & & \\
\hline Universidad Juárez del Estado de Durango & $x$ & & & & & & & & & & $x$ & & & & & & & & & \\
\hline Universidad de Guanaiuato & $\mathrm{x}$ & $x$ & & & & & & & & $x$ & $x$ & & & & & & & & & \\
\hline Universidad Autónoma de Guerrero & & & & & & & & & & & $x$ & & & & & & & & & \\
\hline Universidad Autónoma del Estado de Hidalgo & & $x$ & & & & & $\mathrm{x}$ & & & $\mathrm{x}$ & & & & & & & $x$ & & & \\
\hline Universidad de Guadalajara & $x$ & & & & & & & $\mathrm{x}$ & & $x$ & & & & & $x$ & & & & & \\
\hline Universidad Autónoma del Estado de México & $\mathrm{x}$ & & & & $x$ & & & & $x$ & & & & & & & & & & & \\
\hline Universidad de Michoacana de San Ncolás Hidalgo & $\mathrm{x}$ & & & & & $\mathrm{x}$ & & & $x$ & & & & & & $x$ & & & & & \\
\hline Universidad Autónoma del Estado de Morelos & $\mathrm{x}$ & $x$ & $\mathrm{x}$ & $x$ & & & & & $x$ & $\mathrm{x}$ & $\mathrm{x}$ & & & & & & & & & \\
\hline Universidad Autónoma de Nayarit & & & & & & & & & & $\mathrm{x}$ & & & & & & & & & & \\
\hline Universidad Autónoma de Nuevo León & $\mathrm{x}$ & & & & & & & & & & $\mathrm{x}$ & & & & & & & & & \\
\hline Universidad Autónoma "Benito Juàrez" de Oaxaca & $\mathrm{x}$ & & & & & & & & & & $\mathrm{x}$ & & & & & & & & & \\
\hline Benemérita Universidad Autónoma de Puebla & $\mathrm{x}$ & $x$ & & & & & & & & & $\mathrm{x}$ & & & & & & & & & \\
\hline Universidad Autónoma de Querètaro & $\mathrm{x}$ & & & & & & & & & & $\mathrm{x}$ & & & & $x$ & & & & & \\
\hline Universidad Autónoma de Quintana Roo & $\mathrm{x}$ & & & & & & & & & $\mathrm{x}$ & $x$ & & & & & & & & & \\
\hline Universidad Autónoma de San Luis Potosi & $x$ & $x$ & $\mathrm{x}$ & $\mathrm{x}$ & & $\mathrm{x}$ & $\mathrm{x}$ & $\mathrm{x}$ & & $\mathrm{x}$ & $\mathrm{x}$ & & $x$ & $\mathrm{x}$ & $\mathrm{x}$ & $x$ & $\mathrm{x}$ & $\mathrm{x}$ & & \\
\hline Universidad Autónoma de Sinaloa & & & & & & & & & & & $\mathrm{x}$ & & & & & & & & & \\
\hline Universidad de Sonora & $\mathrm{x}$ & & $x$ & & & $x$ & & & & & & & & $x$ & $\mathrm{x}$ & & & & & \\
\hline Instituto Tecnológico de Sonora & $x$ & & $\mathrm{x}$ & & & & $\mathrm{x}$ & $\mathrm{x}$ & & $\mathrm{x}$ & $\mathrm{x}$ & & $x$ & & & & & & $x$ & $x$ \\
\hline Universidad Juárez Autónoma de Tabasco & $\mathrm{x}$ & $x$ & $\mathrm{x}$ & $\mathrm{x}$ & & & $\mathrm{x}$ & & $\mathrm{x}$ & $\mathrm{x}$ & $\mathrm{x}$ & $x$ & & & & & & & & \\
\hline Universidad Autónoma de Tamaulipas & $\mathrm{x}$ & $x$ & & & & & $x$ & & & & $\mathrm{x}$ & & & & & & & & & \\
\hline Universidad Autónoma de Tlaxcala & $x$ & & & & & $x$ & & & & & $\mathrm{x}$ & & & & & & & & & \\
\hline Universidad Veracruzana & $\mathrm{x}$ & & & & & & $\mathrm{x}$ & & & & $x$ & & & & & & & & & \\
\hline Universidad Autónoma de Yucatán & $x$ & & & $x$ & $x$ & & $x$ & & & & & $x$ & & & $x$ & & & & & \\
\hline Universidad Autónoma de Zacatecas & $x$ & & & & & & & & & $x$ & $x$ & $x$ & & & $\mathrm{x}$ & & & & & \\
\hline $\begin{array}{l}C L=\text { Catálogo en linea } \\
P=\text { Préstamo externo, interno, interbibliotecario } \\
P M A=\text { Préstamo de materiales audiovisuales } \\
A=\text { Préstamo de aulas, àreas de exhibición, } \\
\quad \text { sala de medios eledrónicos. } \\
A D=\text { Apartado de documentos } \\
B=\text { Buzón recep tor de documentos } \\
C=\text { Consulta }\end{array}$ & $\begin{array}{l}\text { AP }=\text { Auto } p r \\
D H I=\text { Desar } \\
R=\text { Referen } \\
B D=\text { Bases } \\
D S \mid=\text { Disem } \\
\mid \mathrm{yD}=\text { Impres } \\
\mathrm{L}=\text { Lectura }\end{array}$ & $\begin{array}{l}\text { ollo } \\
\text { la e } \\
\text { le d } \\
\text { naci } \\
\text { on }\end{array}$ & $\begin{array}{l}\text { no de do } \\
\text { le Habili } \\
\text { linea } \\
\text { tos } \\
\text { on select } \\
\text { digital iza }\end{array}$ & & $\begin{array}{l}\text { ntos } \\
\text { Inform }\end{array}$ & ación & & & & & & $\begin{array}{l}\mathrm{FU}= \\
\mathrm{Lo}= \\
\text { Wifi }= \\
\mathrm{FU}=\end{array}$ & $\begin{array}{l}\text { Forma } \\
\text { Renta } \\
\text { Intern } \\
\text { Forma }\end{array}$ & & $\begin{array}{l}\text { de usu } \\
\text { ckers } \\
\text { lámbr } \\
\text { de usu }\end{array}$ & & & & & \\
\hline
\end{tabular}




\section{Datos de la autora}

Eugenia de los Ángeles Ortega Martínez

Licenciada en Bibliotecología y Estudios en Información; Maestra en Ciencias; Candidata a doctor en Bibliotecología y Estudios en Información por Instituto de Investigaciones Bibliotecológicas en la Universidad Nacional Autónoma de México - UNAM, México.

eugenia.ortega@comunidad.unam.mx

Recibido - Received : 2016-03-02

Aceptado - Accepted: 2016-05-11

\section{(cc) $\mathbf{E Y}$}

This work is licensed under a Creative Commons Attribution 4.0

United States License.

\section{ULIS DD促}

This journal is published by the University Library System of the University of Pittsburgh as part of its D-Scribe Digital Publishing Program and is cosponsored by the University of Pittsburgh Press. 